\title{
Inanspruchnahmebereitschaft und Inanspruchnahmeverhalten von Behandlungen aufgrund psychischer Belastungen bei Patienten der somatischen Rehabilitation
}

\author{
Intention to Use and Use of Treatment Due to Psychological Distress of \\ Patients in Somatic Rehabilitation
}

\author{
Autoren \\ Institut
}

J. Jahed, J. Bengel, H. Baumeister

Abteilung für Rehabilitationspsychologie und Psychotherapie, Institut für Psychologie, Universität Freiburg

\section{Schlüsselwörter \\ psychische Belastung \\ - psychische Störung \\ - medizinische Rehabilitation \\ - Inanspruchnahme}

\section{Key words}

- psychological distress

- mental disorder

- somatic rehabilitation

- health care use

\section{Bibliografie}

DOI http://dx.doi.org/

10.1055/s-0032-1308964

Online-Publikation: 6.6.2012

Rehabilitation 2013;

52: 2-9

(c) Georg Thieme Verlag KG Stuttgart · New York ISSN 0034-3536

\section{Korrespondenzadresse} Dr. Jeanette Jahed Abteilung für Rehabilitationspsychologie und Psychotherapie Institut für Psychologie Universität Freiburg

Engelbergerstraße 41

79106 Freiburg

jeanette.jahed@psychologie. uni-freiburg.de

\section{Zusammenfassung}

$\nabla$

Hintergrund und Zielsetzung: Chronisch körperlich erkrankte Rehabilitanden leiden häufig unter komorbiden psychischen Belastungen und Störungen. Die vorliegende Studie untersucht im Rahmen der Implementierung eines psychodiagnostischen Stufenplans die Inanspruchnahmebereitschaft und das Inanspruchnahmeverhalten von Behandlungen aufgrund psychischer Belastungen.

Methodik: Kohortenstudie mit 6-Monats-Follow-up in 5 orthopädischen, kardiologischen und onkologischen Rehabilitationseinrichtungen $(n=1374)$. Die Erfassung der psychischen Belastung und soziodemografischer Angaben erfolgte mittels Fragebogenerhebung. Fragebogen wurden ebenfalls für die Erhebung der Inanspruchnahmebereitschaft psychotherapeutischer bzw. psychiatrischer Behandlungen und des Inanspruchnahmeverhaltens von Behandlungen aufgrund psychischer Belastungen eingesetzt.

Ergebnisse: Jeder dritte Patient (34\%) war zu Rehabilitationsbeginn psychisch belastet. Eine positive Inanspruchnahmebereitschaft bezüglich psychotherapeutischer bzw. psychiatrischer Behandlung berichteten $79 \%$ der psychisch belasteten Patienten. 6 Monate nach Rehabilitationsende hatten $26 \%$ von den zu Rehabilitationsbeginn belasteten Patienten eine Behandlung aufgrund psychischer Probleme in Anspruch genommen. Eine Nicht-Inanspruchnahme von Behandlungen wurde zu 45\% durch einen nicht vorhandenen Bedarf sowie zu 30\% durch fehlendes Interesse an einer Behandlung trotz wahrgenommenem Bedarf begründet. Weitere $25 \%$ dachten über eine Behandlung nach bzw. waren auf der Suche nach einem passenden Angebot.

Schlussfolgerung: Psychisch belastete Rehabilitanden weisen eine hohe Inanspruchnahmebereitschaft auf. Das Inanspruchnahmeverhalten post-rehabilitativer, die psychischen Belastungen betreffender Behandlungsmaßnahmen fällt hin-

\section{Abstract \\ $\nabla$}

Background and objectives: Somatically ill patients in somatic rehabilitation often show comorbid psychological distress and mental disorders. In the framework of the implementation of a stepwise psychodiagnostic procedure, the present study investigated the intention to use and the use of treatment due to psychological distress.

Methodology: Cohort study with a 6-months follow-up in 5 orthopedic, cardiologic and oncologic inpatient rehabilitation clinics $(n=1374)$. Psychological distress, sociodemographic data, intention to use psychotherapeutic or psychiatric treatments and the use of health care offers due to psychological distress were assessed by means of questionnaires.

Results: Every third patient (34\%) was psychologically distressed at the beginning of rehabilitation. A positive intention to use psychotherapeutic or psychiatric treatment was reported by $79 \%$ of the psychologically distressed patients. 6 months after the end of rehabilitation, $26 \%$ of the patients who were psychologically distressed at the beginning of rehabilitation, had received treatment for mental health reasons. Reasons for non-utilization of treatment included lack of subjective need (45\%) and lack of interest in treatment despite a perceived need (30\%). Further 25\% were considering treatment or were seeking appropriate treatment.

Conclusions: Psychologically distressed rehabilitation patients show a high intention to undergo treatment. However, they show a clearly diminished use of post-rehabilitative treatments due to mental health problems. Hence, the promotion of motivation as well as the management of treatment barriers for improving post-rehabilitative use in psychologically distressed rehabilitants are of particular importance. 
gegen deutlich geringer aus. Ein Ziel psychologischer Maßnahmen in der Rehabilitation sollte folglich die Motivationsförderung und das Barrierenmanagement zur Verbesserung des postrehabilitativen Inanspruchnahmeverhaltens psychisch belasteter Rehabilitanden sein.

\section{Einleitung}

Chronisch körperlich erkrankte Patienten leiden häufig unter komorbiden psychischen Störungen [1-6]. In der medizinischen Rehabilitation weisen 38\% der Patienten eine psychische Belastung und jeder fünfte Patient eine psychische Störung auf [7]. Komorbide psychische Störungen sind mit erhöhten Morbiditäts- und Mortalitätsraten [8,9], erhöhten Versorgungskosten $[10,11]$ sowie niedriger Compliance $[12,13]$ und Lebensqualität $[9,14]$ assoziiert. Gleichzeitig zeigt sich trotz des biopsychosozialen Ansatzes der medizinischen Rehabilitation eine unzureichende Entdeckungsrate psychischer Störungen [15-17]. Aufgrund dieser Erkenntnisse wurde 2004 von einer Expertenrunde, bestehend aus Rehabilitationswissenschaftlern, Leistungsträgern und Leistungserbringern, ein psychodiagnostisch abgestuftes Vorgehen für die somatische Rehabilitation empfohlen [18]. Im Rahmen der DIBpS-Studie (Diagnostik, Indikation und

Tab. 1 Psychodiagnostischer Stufenplan.

\begin{tabular}{|c|c|}
\hline Stufe & Beschreibung \\
\hline I. & $\begin{array}{l}\text { Eingangsscreening } \\
\text { z. B. mittels PHQ- } 9 \text { oder HADS }\end{array}$ \\
\hline II. & $\begin{array}{l}\text { vertiefte psychodiagnostische Untersuchung } \\
\text { z. B. mittels Screeningfragen und } \\
\text { z. B. mittels Internationaler Diagnose-Checklisten für ICD-10 }\end{array}$ \\
\hline III. & $\begin{array}{l}\text { Indikationsstellung und Behandlung } \\
\text { für spezifische psychologische, psychotherapeutische oder } \\
\text { psychopharmakologische Behandlung }\end{array}$ \\
\hline IV. & $\begin{array}{l}\text { Dokumentation } \\
\text { psychischer Belastungen und Störungen im ärztlichen Entlas- } \\
\text { sungsbericht } \\
\text { Aussprechen einer Behandlungsempfehlung für die post-rehabi- } \\
\text { litative Versorgung }\end{array}$ \\
\hline
\end{tabular}

Behandlung von psychischen Störungen in der medizinischen Rehabilitation) wurde ein psychodiagnostischer Stufenplan in Anlehnung an die Speyerer Empfehlungen [18] entwickelt und seine Implementierung in 13 Rehabilitationskliniken evaluiert [19-21] ( Tab. 1).

Neben einer adäquaten psychodiagnostischen Routine ist die Bereitschaft der Rehabilitanden, die auf die Psychodiagnostik folgenden Behandlungsempfehlungen auch umzusetzen, eine weitere wichtige Voraussetzung zur notwendigen Optimierung der Versorgung komorbid psychisch belasteter Patienten [22-25]. Ein Teilziel der DIBpS-Studie war entsprechend die Untersuchung der Inanspruchnahmebereitschaft sowie des Inanspruchnahmeverhaltens von Behandlungen aufgrund psychischer Belastungen. Folgende 2 Fragestellungen wurden untersucht:

- Wie hoch ist die Inanspruchnahmebereitschaft für psychotherapeutische bzw. psychiatrische Versorgungsangebote von somatisch erkrankten Rehabilitanden mit komorbiden psychischen Belastungen oder Störungen?

- Wie hoch ist die Inanspruchnahme von Behandlungsmaßnahmen 6 Monate nach der Rehabilitation bei zu Rehabilitationsbeginn psychisch belasteten Patienten der somatischen Rehabilitation?

\section{Methodik}

\section{Studiendesign}

Im Rahmen der Studie wurde in 5 Rehabilitationseinrichtungen mit den Indikationsbereichen Orthopädie, Kardiologie und Onkologie über einen 5-monatigen Zeitraum ergänzend zur Implementierung des psychodiagnostischen Stufenplans eine schriftliche Befragung aller Patienten durchgeführt. Anhand eines 2-stufigen Untersuchungsplans ( $\bullet$ Abb. 1) wurden Patienten in einem ersten Schritt zu Rehabilitationsbeginn mittels Fragebogen hinsichtlich psychischer Belastungen und Inanspruchnahmebereitschaft psychotherapeutischer bzw. psychiatrischer Behandlungsmaßnahmen, bisheriger Inanspruchnahme sowie soziodemografischer Angaben befragt $(n=1374)$. In einem zweiten Schritt wurden die Patienten, bei denen zu Rehabilitationsbeginn eine psychische Belastung vorlag, 6 Monate nach Ende der Rehabilitation erneut hinsichtlich psychischer Belastungen

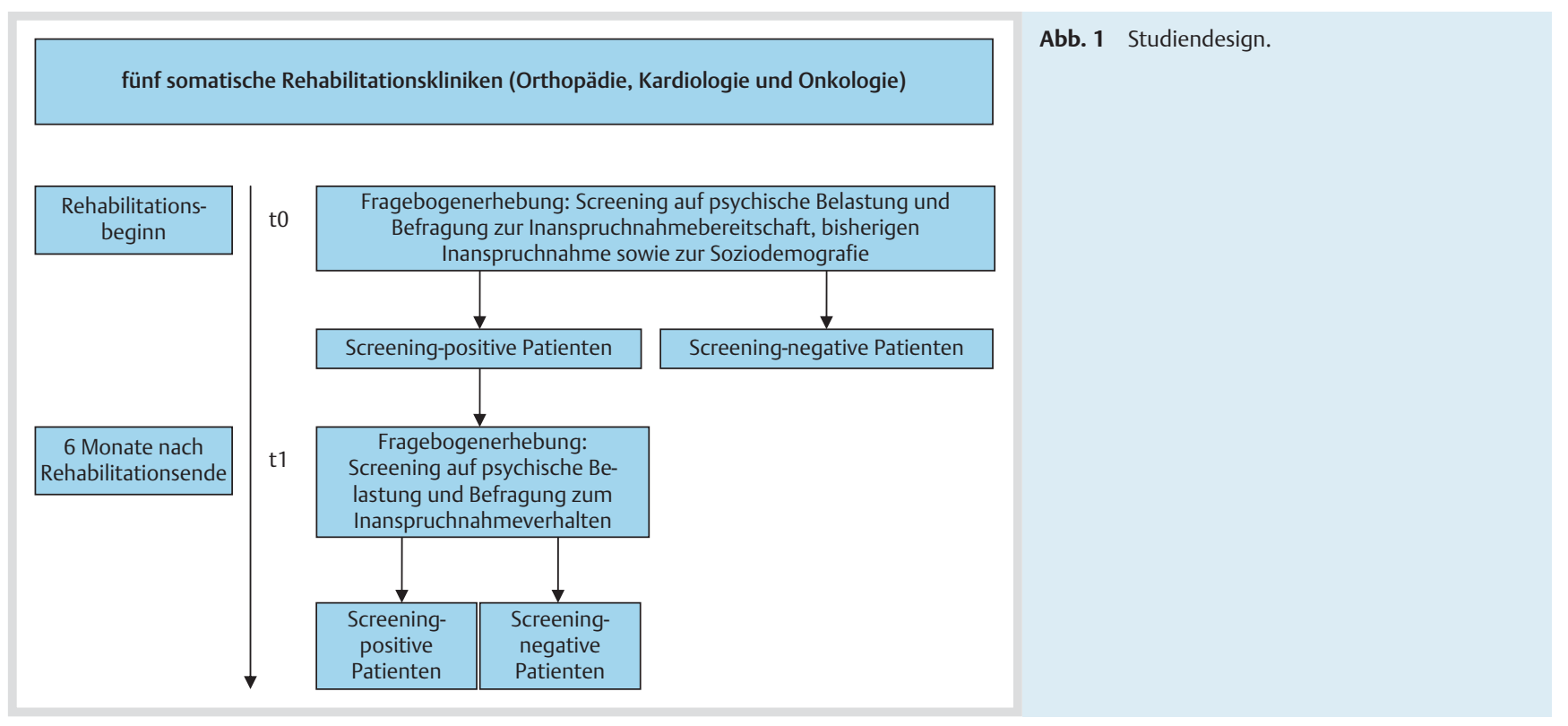


sowie der Inanspruchnahme von Behandlungen aufgrund von psychischen Belastungen befragt $(n=261)$.

\section{Datenerhebung}

Soziodemografie

$\mathrm{Zu}$ Rehabilitationsbeginn wurden folgende soziodemografische Angaben erhoben: Geschlecht, Alter, Staatsangehörigkeit, Familienstand und Kinderzahl. Zudem wurden Fragen zum sozioökonomischen Status gestellt, die sich auf den höchsten erreichten Schulabschluss, eine abgeschlossene Berufsausbildung sowie auf den derzeitigen Erwerbstätigkeitsstatus bzw. Rentenantragsstatus bezogen.

\section{Psychische Belastung}

Zur Erfassung der psychischen Belastung wurde der ScreeningFragebogen Patient Health Questionnaire (PHQ-9) [26] bzw. Hospital Anxiety and Depression Scale (HADS) [27] verwendet. Die teilnehmenden Kliniken konnten zwischen den beiden Screening-Fragebogen wählen. Beide Instrumente weisen gute Gütekriterien auf und eignen sich zur validen Erfassung irgendeiner psychischen Störung $[28,29]$. Der Selbstbeurteilungsfragebogen PHQ-9 stellt mit 9 Items zur Depressivität und einem Score-Range von 0-27 eine Kurzversion des PHQ-D dar. Der optimale Cut-off zur Vorhersage psychischer Störungen beträgt $\geq 10[26,28]$. Die HADS ist ein Selbstbeurteilungsinstrument und erfasst anhand von je 7 Items und einem Score-Range von 0-42 Angst und Depressivität. Als optimaler Cut-off gilt für die Angstskala ein Wert von $\geq 11$ und für die Depressionsskala $\geq 9$ [27]. Bei Überschreitung des jeweiligen Cut-off-Wertes wurden Patienten als psychisch belastet eingestuft.

\section{Inanspruchnahmebereitschaft}

Die Inanspruchnahmebereitschaft wurde mit einem Item mit einer 4-stufigen Antwortmöglichkeit erhoben: „Angenommen, Ihre Behandler in der Rehabilitationseinrichtung stellen bei Ihnen seelische Probleme (z.B. Angststörung, Depression) fest, würden Sie psychotherapeutische bzw. psychiatrische (nervenärztliche) Hilfe in Anspruch nehmen?“ Die Antwortmöglichkeiten lauteten „Ja“, „Höchstwahrscheinlich schon“, „Eher nicht“ und „Nein“.

\section{Inanspruchnahmeverhalten}

Die Inanspruchnahme von Behandlungen vor der Rehabilitation wurde mit der Frage „Ich befinde mich derzeit in psychotherapeutischer bzw. psychiatrischer (nervenärztlicher) Behandlung“ mit den Antwortmöglichkeiten „Nein“ und „Ja“ erhoben. Die Inanspruchnahme einer Behandlung wegen psychischer Probleme nach der Rehabilitation wurde mit der Frage erhoben: „Waren Sie innerhalb der letzten 6 Monate wegen seelischer Probleme in Behandlung?“ Neben den Antwortmöglichkeiten „Nein“ und „Ja, stationär“ wurde unter der weiteren Option „Ja, ambulant“ erfragt, welche Berufsgruppe aufgrund psychischer Probleme in Anspruch genommen wurde (Hausarzt, Psychiater, Psychotherapeut, anderer Arzt oder sonstige Berufsgruppe). Innerhalb der Antwortmöglichkeiten „Nein“ wurde differenziert zwischen den 4 Möglichkeiten „Ich hatte bisher keinen Bedarf“, „Ich fühle mich zwar seelisch belastet, habe aber kein Interesse an einer psychotherapeutischen oder psychiatrischen (nervenärztlichen) Behandlung“, „Aber ich denke darüber nach, eine psychotherapeutische oder psychiatrische Behandlung zu beginnen“ und „Aber zurzeit bin ich auf der Suche nach einem passenden Angebot".

\section{Datenanalyse}

Anhand der Screeningergebnisse wurde eine Dichotomisierung in psychisch unbelastete (Screening-negative) und psychisch belastete (Screening-positive) Patienten basierend auf den zuvor genannten Cut-off-Werten vorgenommen. Die Inanspruchnahmebereitschaft und das Inanspruchnahmeverhalten der psychisch belasteten Rehabilitanden wird in Prozent berichtet. Des Weiteren wurde die Assoziation zwischen Inanspruchnahmebereitschaft und dem postrehabilitativen Inanspruchnahmeverhalten sowie die Assoziation dieser Inanspruchnahmevariablen mit bedeutsamen Drittvariablen (Alter, Geschlecht, Bildung und Familienstand [30-33]) mittels Chi-Quadrat-Tests getestet. Die statistische Auswertung erfolgte mittels PASW Statistics Version 18 (SPSS).

\section{Ergebnisse \\ $\nabla$}

Soziodemografische Daten und psychische Belastung

Die Gesamtstichprobe $(\mathrm{n}=1374)$ war zu Beginn der Rehabilitation (t0) im Durchschnitt 58,3 Jahre alt (Mittelwert der Jahresstatistik der beteiligten Kliniken: 61,7 Jahre) und zu 50,7\% weiblich (Jahresstatistik: 54,2\%); 34,4\% der Rehabilitanden waren psychisch belastet. 6 Monate nach Ende der Rehabilitation (t1) belief sich die Stichprobengröße auf 261 Patienten (Rücklauf: $55 \%$ aller zu t0 psychisch Belasteten) mit einem Durchschnittsalter von 55,3 Jahren und einem Geschlechterverhältnis ( $F$ : M) von 55,6 : 44,4\%. 55,2\% der zu Rehabilitationsbeginn psychisch belasteten Rehabilitanden waren auch 6 Monate nach Ende der Rehabilitation psychisch belastet ( $\bullet$ Tab. 2).

\section{Inanspruchnahmebereitschaft}

- Abb. 2 zeigt die Inanspruchnahmebereitschaft einer psychotherapeutischen oder psychiatrischen Behandlung bei Patienten, die zu Rehabilitationsbeginn psychisch belastet waren. Wird die Inanspruchnahmebereitschaft dichotomisiert in eine positive („Ja“ und „Höchstwahrscheinlich schon“) und eine negative („Nein“ und „Eher nicht“) Bereitschaft, berichteten 79,4\% eine positive Inanspruchnahmebereitschaft.

In Bezug auf mögliche Drittvariablen (Alter, Geschlecht, Bildung oder Familienstand) auf die Inanspruchnahmebereitschaft zeigte sich nur für das Alter ein signifikanter Zusammenhang $(n=457$; $\mathrm{p}<0,001$ ). Psychisch belastete Patienten unter 65 Jahren waren häufiger bereit, eine Behandlung in Anspruch zu nehmen, als Patienten über 65 Jahre (83,4\% vs. 67,5\%).

\section{Inanspruchnahmeverhalten}

Vor Beginn der Rehabilitation waren 11,8\% der zu t0 psychisch belasteten Patienten in psychotherapeutischer bzw. psychiatrischer Behandlung. Von den katamnestisch befragten Rehabilitanden $(n=261)$ gaben $26,1 \%$ an, sich aufgrund psychischer Belastungen innerhalb der letzten 6 Monate in Behandlung befunden zu haben bzw. aktuell noch zu befinden. Von den zu t0 und t1 psychisch belasteten Rehabilitanden $(n=144)$ berichteten $37,5 \%$, in den vergangenen 6 Monaten wegen psychischer Probleme in Behandlung gewesen zu sein. Bei der Gruppe der zu t1 nicht mehr Belasteten $(n=117)$ trifft dies auf $12,0 \% \mathrm{zu}$. Bis auf 2 Fälle mit stationärer Behandlung wurden aufgrund psychischer Probleme ambulante Behandlungsdienste (Mehrfachantworten möglich) in Anspruch genommen. Am häufigsten wurde ein Psychotherapeut, gefolgt von einem Psychiater und einem Hausarzt aufgesucht ( $\bullet$ Abb. 3). 
Tab. 2 Soziodemografie und psychische Belastung der Stichprobe zu Rehabilitationsbeginn (t0) sowie zum Katamnese-Zeitpunkt (t1).

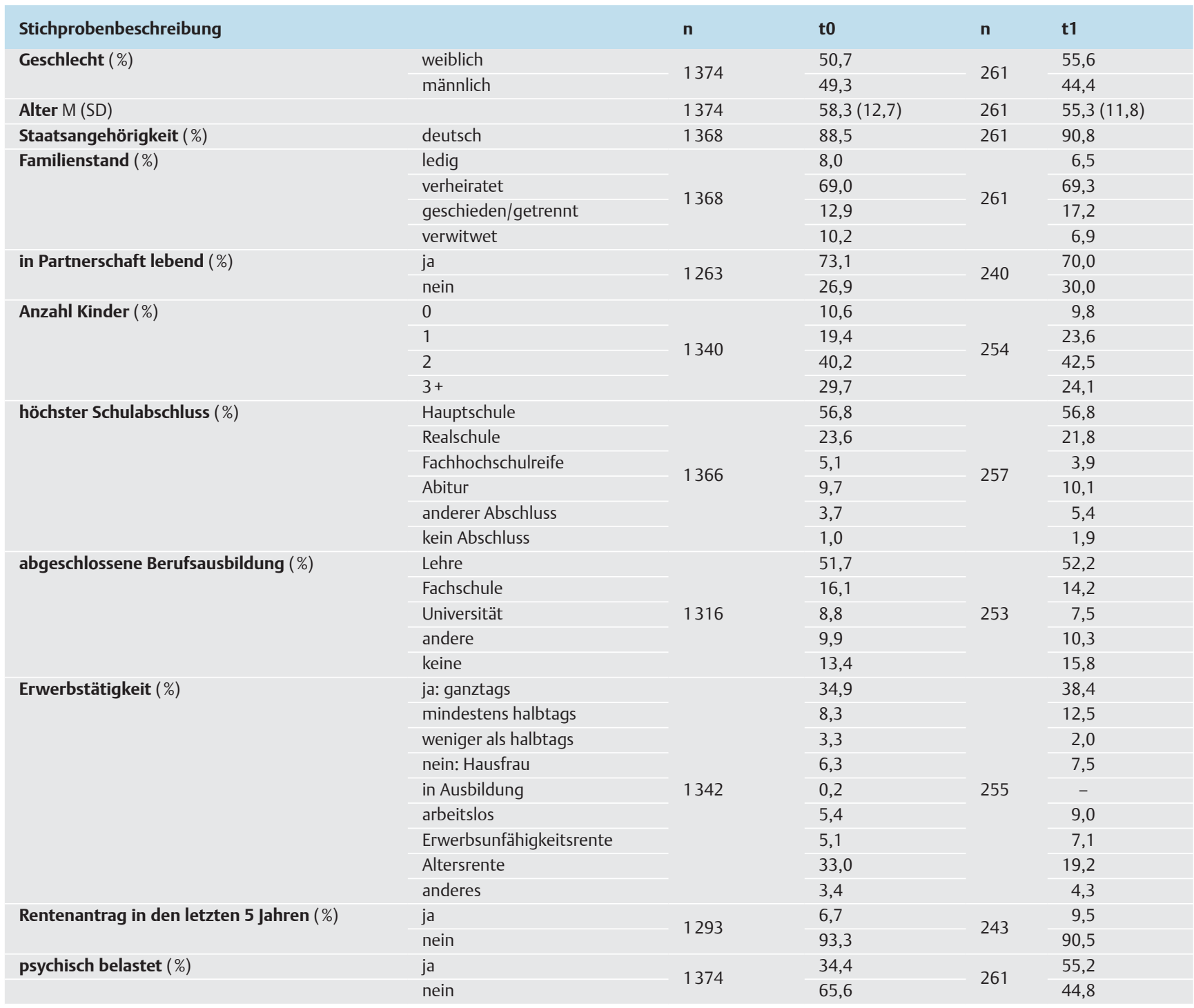

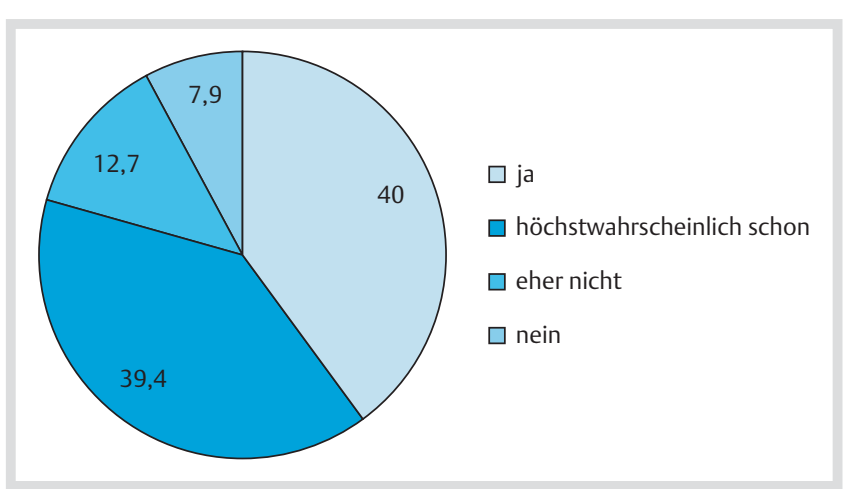

Abb. 2 Inanspruchnahmebereitschaft psychotherapeutischer oder psychiatrischer Behandlungen bei zu t0 psychisch belasteten Patienten in Prozent $(n=457)$.

In den zurückliegenden 6 Monaten hatten 73,9\% aller zu t1 Befragten und 62,5\% der auch zu t1 noch psychisch belasteten Rehabilitanden keine Behandlung in Anspruch genommen. Hiervon dachten $17,1 \%$ respektive $31,6 \%$ über eine Behandlung nach und weitere $8,1 \%$ bzw. 12,3\% waren auf der Suche nach einem passenden Angebot. Keinen Bedarf sahen 44,7\% aller zu t1 Befragten und $17,5 \%$ der zu t1 psychisch belasteten Rehabilitanden. Trotz wahrgenommenen Bedarfs kein Interesse an einer Behandlung zu haben gaben $30,1 \%$ sowie $38,6 \%$ an ( $\bullet$ Abb. 4 ). Zwischen der Inanspruchnahme einer Behandlung nach der Rehabilitation und dem Geschlecht, der Bildung oder dem Familienstand fanden sich keine Zusammenhänge. Ein signifikanter Effekt zeigte sich hingegen für den Zusammenhang zwischen der Inanspruchnahme und dem Alter. So wurde von den unter 65-Jährigen signifikant häufiger eine Behandlung aufgrund von psychischen Problemen in Anspruch genommen als von den über 65-Jährigen (28,8\% vs. 15,1\%; $n=261 ; p=0,021)$. Dieses Ergebnis zeigte sich in ausgeprägterer Form auch für die Subgruppe der zu 11 noch psychisch belasteten Rehabilitanden (44,1\% vs. $15,2 \% ; n=144 ; p=0,003$ ).

Ein signifikanter Zusammenhang bestand zwischen der Inanspruchnahmebereitschaft der zu t0 psychisch belasteten Rehabilitanden und der postrehabilitativen Inanspruchnahme $(n=256$; $\mathrm{p}=0,001$ ). Eine Behandlung in Anspruch genommen zu haben berichteten 33,6\% (,ja“) respektive 26,9\% („höchstwahrscheinlich schon“) der Rehabilitanden mit einer eher positiven Inanspruchnahmebereitschaft sowie 9,1\% („eher nicht“) und $0 \%$ 


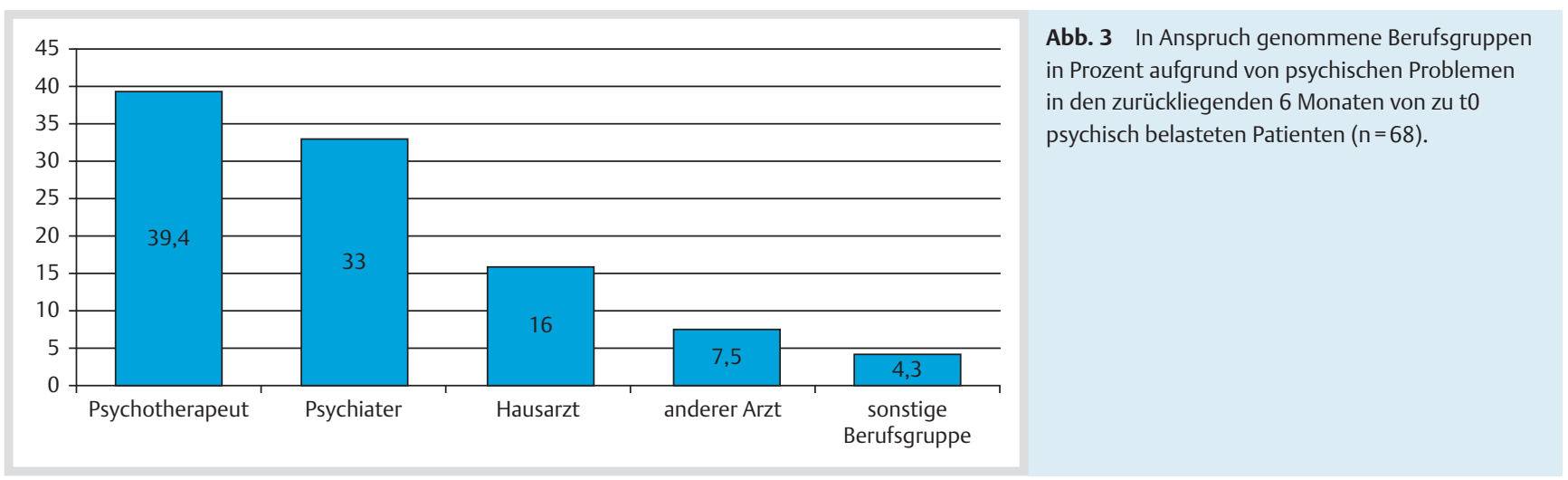

(„nein“) der Rehabilitanden mit einer eher negativen Inanspruchnahmebereitschaft.

\section{Diskussion}

$\nabla$

Bezüglich des Durchschnittsalters und der Geschlechtsverteilung entspricht die untersuchte Stichprobe der Jahresstatistik der beteiligten Kliniken. Zu Beginn der Rehabilitation war jeder dritte Patient (34,4\%) psychisch belastet. Dies ist vergleichbar mit der Prävalenz psychischer Belastungen (38\%) bei Patienten der medizinischen Rehabilitation [7].

$\mathrm{Zu}$ Rehabilitationsbeginn bestand eine hohe Bereitschaft zur Inanspruchnahme eines psychotherapeutischen oder psychiatrischen Behandlungsangebots, falls eine entsprechende Belastung festgestellt werden würde. Rund $80 \%$ der psychisch belasteten Rehabilitanden gaben an bereit zu sein, ein psychotherapeutisches oder psychiatrisches Angebot in Anspruch zu nehmen (,ja“ bzw. „höchstwahrscheinlich schon“). Hahn et al. [34] berichten nach einer Arztempfehlung ähnlich hohe Raten der Inanspruchnahmebereitschaft (75\%) bei psychisch belasteten Patienten. Ohne Empfehlung wären noch 60\% der psychisch Belasteten an einer Behandlung interessiert. Es ist anzunehmen, dass Patienten gerade zu Beginn der Rehabilitation aufgrund eines schnellen Genesungswunsches, eines Neuigkeitswertes oder aber auch aufgrund einer Überforderung besonders offen für diverse Behandlungsangebote sind. Dies kann sich ggf. im Laufe der Rehabilitation unter anderem vor dem Hintergrund spezifischeren Wissens oder erster professioneller Kontakte ändern. Nach Abklärung eines möglichen Behandlungsbedarfs stehen zur weiteren Förderung der Behandlungsmotivation eine Vielzahl von Techniken und Interventionen zur Verfügung [23,34-36]. Inhaltlich können sich die Interventionen beispielsweise auf die Steigerung der Risiko- und Selbstwirksamkeitswahrnehmung sowie auf konkrete Hilfestellungen in Bezug auf die Suche nach einem passenden post-rehabilitativen Behandlungsangebot und die Bearbeitung bestehender Behandlungshindernisse im Sinne des Barrierenmanagements beziehen. Hierbei sollte der Patient auch auf die derzeit häufig anzutreffende ambulante Versorgungssituation mit langen Wartezeiten für einen Psychotherapieplatz hingewiesen werden, um Frustration entgegenwirken zu können.

Von den zu Rehabilitationsbeginn psychisch belasteten Patienten waren zuvor knapp $12 \%$ in psychotherapeutischer bzw. psychiatrischer Behandlung gewesen. Mit 26,1\% nahmen mehr als doppelt so viele Patienten eine post-rehabilitative Behandlung in Anspruch. Dies entspricht in etwa den Ergebnissen zur Ver- sorgung von psychischen Störungen in der Allgemeinbevölkerung. Wittchen und Jacobi [37] berichten bei einer Jahresprävalenz von 32 \% für eine oder mehrere psychische Störungen in der erwachsenen deutschen Bevölkerung eine Inanspruchnahmerate der Betroffenen von 36\%. Allerdings hatten davon lediglich $10 \%$ eine nach wissenschaftlichen Kriterien adäquate Therapie erhalten. Die Autoren sehen darin eine gravierende Unterversorgung von Personen mit psychischen Störungen. Zu beachten gilt bei diesem Vergleich, dass mittels Screeninginstrumenten ermittelte psychische Belastungen nur einen Hinweis auf behandlungsbedürftige psychische Störungen bieten. Der in den Kliniken eingeführte psychodiagnostische Stufenplan ( $\bullet$ Tab. 1) trägt diesem Sachverhalt Rechnung, indem aufbauend auf einem Screening-positiven Befund eine vertiefte psychodiagnostische Abklärung empfohlen wird. Im Anschluss sollte bei Bedarf eine Indikationsstellung für spezifische psychologische, psychotherapeutische oder psychopharmakologische Behandlungsmaßnahmen ausgesprochen werden. Hierbei ist zu beachten, dass die Indikationsstellung gemeinsam mit dem Patienten stattfinden sollte. So können der individuelle Behandlungsbedarf festgestellt und ggf. Maßnahmen zur Motivationsförderung durchgeführt werden. Eine Behandlung von psychischen Belastungen und Störungen ist nicht immer zwingend notwendig, beispielsweise wenn genügend eigene Ressourcen (z.B. soziale Unterstützung) vorhanden sind und dadurch mit einer Verbesserung bzw. Behebung der Symptomatik zu rechnen ist. Gleiches gilt, wenn die psychische Belastung als subklinisch oder transient im Sinne einer Belastungsreaktion eingestuft wird [38,39]. Der Vergleich der zu Rehabilitationsbeginn psychisch belasteten Rehabilitanden mit und ohne psychische Belastungen 6 Monate nach der Rehabilitation ( $\bullet$ Abb. 4 ) deutet auf einen wünschenswerten Selbstselektionseffekt der Patienten hin. Während 44,7\% aller katamnestisch befragten Rehabilitanden keinen Behandlungsbedarf für sich angaben, traf dies nur auf $17,5 \%$ der weiterhin psychisch belasteten Rehabilitanden zu. Die Ergebnisse verdeutlichen jedoch auch die Notwendigkeit einer erneuten psychodiagnostischen Abklärung nach der Rehabilitation im Sinne eines aktiven Monitorings, um chronisch-progredient verlaufende psychische Belastungen und Störungen frühzeitig zu erkennen und vorliegende Hindernisse und Vorbehalte gegenüber entsprechenden Behandlungsmaßnahmen aufzulösen.

Diejenigen Patienten, die eine Behandlung wegen psychischer Probleme in Anspruch nahmen, taten dies überwiegend bei einem Psychotherapeuten oder einem Psychiater. Dieses Ergebnis steht im Widerspruch zu Daten zur Versorgung psychischer Störungen in der Allgemeinbevölkerung $[37,40]$, in der sich die Hausarztversorgung als zentral erwies. Eine Erklärung für diese 

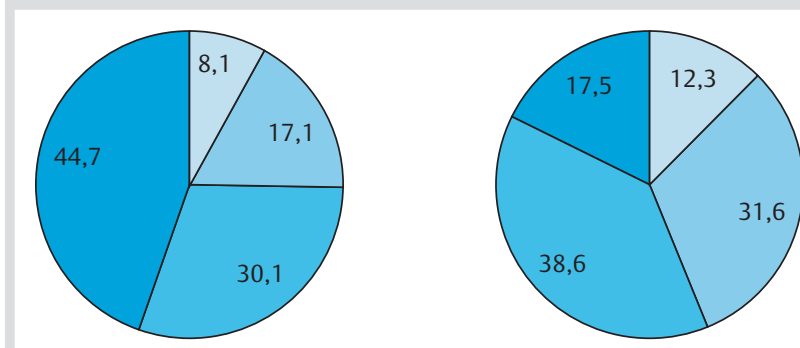

$\square$ auf der Suche

$\square$ denke über Behandlung nach

$\square$ trotz Bedarf kein Behandlungsinteresse

$\square$ kein Behandlungsbedarf
Abb. 4 Gründe für eine bisherige Nicht-Inanspruchnahme von Behandlungsmaßnahmen in den zurückliegenden 6 Monaten nach Rehabilitationsende von allen t1-Befragten ( $n=123$; links) sowie von den auch noch zu $\mathrm{t} 1$ psychisch belasteten Patienten ( $n=57$; rechts) in Prozent.
Differenz könnte sein, dass der Kontakt mit dem psychologischen Dienst im Rahmen des Rehabilitationsaufenthalts die Wahrscheinlichkeit der Inanspruchnahme eines Facharztes oder Psychotherapeuten erhöht. Ebenso könnte es sein, dass die Differenzierungsfähigkeit zwischen den einzelnen Behandlergruppen eingeschränkt ist und die Ergebnisse aufgrund dessen verzerrt sind.

Fast die Hälfte der 6 Monate nach ihrem Rehabilitationsaufenthalt weiterhin noch psychisch belasteten Rehabilitanden gaben als Grund für ihre bisherige Nicht-Inanspruchnahme an, auf der Suche nach einem passenden Behandlungsangebot zu sein oder über eine Behandlung nachzudenken. Die genannten konkreten Hilfestellungen in Bezug auf die Suche nach einem passenden post-rehabilitativen Behandlungsangebot sowie einem möglichst individualisierten Barrierenmanagement könnten bedeutsame Hilfestellungen zur Umsetzung der Behandlungsabsicht für diese Patienten darstellen.

Hiervon abzugrenzen sind Patienten mit selbstwahrgenommenem Behandlungsbedarf, aber fehlendem Behandlungsinteresse. Dies traf auf 38,6\% aller auch im Follow-up noch psychisch belasteten Rehabilitanden zu. Bei diesen Patienten steht die Exploration und Modifikation der jeweiligen Hinderungsgründe im Fokus motivationsfördernder Maßnahmen [23,34,36]. Hinderungsgründe könnten beispielsweise ein somatisches Krankheitsverständnis, Vorbehalte aufgrund von Stigmatisierungsängste, Zeitmangel, ein gering eingeschätzter Behandlungserfolg sowie negative Vorerfahrungen sein.

Knapp ein Fünftel der auch im Follow-up noch psychisch belasteten Rehabilitanden sah keinen Behandlungsbedarf. Gründe hierfür könnten einerseits ein tatsächlich nicht gegebener Behandlungsbedarf aufgrund von z.B. ausreichender eigener Bewältigungsmöglichkeiten oder einem falsch-positiven Screeningergebnis sein. Andererseits könnte eine nicht ausreichende Krankheitseinsicht dafür verantwortlich sein. Ein fehlendes Problembewusstsein kann beispielsweise darin begründet sein, dass Patienten die negativen Konsequenzen ihres Verhaltens nicht mit ihrer Erkrankung in Verbindung bringen (z. B. Alkoholabhängigkeit und Beziehungsproblematik), ein somatisches Erklärungsmodell ihrer Symptomatik aufweisen oder ihre Störung nicht als Problem betrachten [34]. Ziel psychologischer Behandlungsmaßnahmen bei diesen Patienten ist es, ein Problembewusstsein zu erarbeiten, indem man z.B. dem Patienten das Wissen vermittelt, um seine Erkrankung neu einordnen zu können, sowie eine „Motivation auf Zeit“ generiert („Was schadet es Ihnen, wenn Sie es einfach mal versuchen?“) $[34,36]$.

Sowohl für die Inanspruchnahmebereitschaft als auch für das Inanspruchnahmeverhalten zeigte sich für das Alter ein signifikanter Zusammenhang. Damit bestätigen sich Befunde einer geringeren Inanspruchnahmebereitschaft bzw. eines geringen Inanspruchnahmeverhaltens psychotherapeutischer bzw. psychiatrischer Leistungen bei älteren Patienten mit psychischen Belas- tungen und Störungen [41-44]. Als mögliche Ursachen werden trotz des Nachweises der Wirksamkeit psychotherapeutischer Verfahren für eine Vielzahl psychischer Störungen auch bei älteren Patienten [45-48] die Therapieeinstellung älterer Patienten sowie die Behandlungsbereitschaft der Therapeuten genannt $[49,50]$. Zusätzlich können limitierende Faktoren durch das Versorgungssystem beispielsweise aufgrund einer geringen Versorgungsdichte, einer schlechten Erreichbarkeit und der NichtSpezialisierung auf gerontologische Themen auftreten $[49,50]$. Demnach ist für diese Patientengruppe die Aufklärung über und die Motivation zu verschiedenen Behandlungsoptionen von besonderer Bedeutung. Bezüglich des Geschlechts ließ sich kein Zusammenhang feststellen. Dieses Ergebnis reiht sich in die inkonsistenten Befunde früherer Studien ein, die teils eine höhere Inanspruchnahme psychisch belasteter Frauen im Vergleich zu psychisch belasteten Männern aufzeigten [30,41,51-53] und teils keinen Zusammenhang berichteten [33,54]. Zwischen einem höheren Schulabschluss und der Inanspruchnahme einer Behandlung ergab sich kein Zusammenhang. Dies zeigt sich auch in verschiedenen früheren Studien $[32,33,55]$, während in anderen Studien höhere Bildung mit höherer Inanspruchnahme einherging [31,53]. Bezüglich des Familienstandes und der Inanspruchnahme zeigte sich kein signifikanter Zusammenhang. Die Forschungsliteratur ist auch hierzu inkonsistent. Zum einen gibt es Hinweise auf eine höhere Inanspruchnahme bei Alleinlebenden (z.B. geschieden, verwitwet) [30,44,53], zum anderen konnten keine Zusammenhänge festgestellt werden [33].

Bei der Interpretation der Ergebnisse ist zu beachten, dass es durch den Einsatz der verwendeten Screeninginstrumente zu methodischen Einschränkungen gekommen sein könnte. Da die Kliniken im Zuge einer besseren Akzeptanz der Implementierung des psychodiagnostischen Stufenplans zwischen dem Einsatz des PHQ-9 und der HADS wählen konnten ( $\bullet$ Tab. 1 ), wurden die Patienten der 5 Kliniken nicht einheitlich mit demselben Screener untersucht. Jedoch wurden bei der Ergebnisdarstellung die Screening-positiven bzw. -negativen Patienten jeweils instrumentenübergreifend zusammengefasst. Vor dem Hintergrund, dass beide Verfahren gute Gütekriterien aufweisen [28,29], die empfohlenen Cut-off-Werte verwendet wurden [26-28] und die gezeigte Prävalenzrate psychischer Belastungen vergleichbar mit der in einer repräsentativen epidemiologischen Studie [7] ist, scheint es zu keiner bedeutsamen Verzerrung gekommen zu sein. Eine mögliche Ergebnisverzerrung in Bezug auf die Fragestellung zu den spezifischen Gründen einer bisherigen NichtInanspruchnahme ist aufgrund von fehlenden Werten nicht auszuschließen.

Abschließend dürften die in der vorliegenden Studie aufgezeigten Inanspruchnahmeraten die durchschnittliche Inanspruchnahmerate aller Rehabilitanden der medizinischen Rehabilitation im Sinne eines „Best Case Scenario“ überschätzen. Die Erfassung des Inanspruchnahmeverhaltens erfolgte nach der Imple- 
mentierung des psychodiagnostischen Stufenplans, dessen Ziel es ist, die psychodiagnostische Routine in Rehabilitationskliniken zu optimieren und zu einer verbesserten Dokumentation einschließlich entsprechender post-rehabilitativer Behandlungsempfehlungen beizutragen. Die positive Evaluation des Stufenplans [19-21] könnte dafür sprechen, dass es auch patientenseitig zu einer verbesserten Versorgung komorbid belasteter Patienten im Sinne einer erhöhten Inanspruchnahme post-rehabilitativer Behandlungsangebote kam.

\section{Kernbotschaft}

Bei entsprechender Indikation sollten Maßnahmen zur Förderung der Behandlungsmotivation sowie zum Barrieremanagement während und nach der Rehabilitation eingesetzt werden, um das Inanspruchnahmeverhalten bei psychischen Belastungen und Störungen weiter zu steigern. Insbesondere der bedeutsame Anteil von Patienten, der einer Behandlung gegenüber noch unentschlossen ist, könnte hiervon substantiell profitieren.

\section{Danksagung}

\section{$\nabla$}

Wir danken den teilnehmenden Patienten und Rehabilitationseinrichtungen der Pilot- und Hauptstudie: Asklepios Klinik Triberg, Triberg; Fachklinik Waldbronn, Waldbronn; Mooswaldklinik, Freiburg; Klinik Baden, Bad Krozingen; Klinik im Hofgarten, Bad Waldsee; REGIO-RehaTagesklinik, Freiburg; Rehabilitationsklinik Höhenblick, Baden-Baden; Sankt Rochus Kliniken, Bad Schönborn; Schwarzwaldklinik, Bad Krozingen; Städtische Rehakliniken, Bad Waldsee; Theresienklinik, Bad Krozingen; Waldeck Klinik, Bad Dürrheim; Winkelwaldklinik Nordrach, Nordrach.

\section{Förderung}

$\nabla$

Dieses Forschungsprojekt wird durch Mittel der Deutschen Rentenversicherung Bund gefördert (Förderkennzeichen: 0423/ 00-40-64-50-04).

Interessenkonflikt: Es bestehen keine Interessenkonflikte.

\section{Literatur}

1 Baumeister H, Härter M. Mental disorders in patients with obesity in comparison with healthy probands. International Journal of Obesity 2007; 31: 1155-1164

2 Härter M, Baumeister $H$, Reuter $K$ et al. Increased 12-month prevalence rates of mental disorders in patients with chronic somatic diseases. Psychotherapy and Psychosomatics 2007; 76: 354-360

3 He Y, Zhang M, Lin EH et al. Mental disorders among persons with arthritis: results from the World Mental Health Surveys. Psychological Medicine 2008; 38: 1639-1650

4 Lin EH, von Korff M on behalf of the WHO WMH Survey Consortium. Mental Disorders among Persons with Diabetes - results from the World Mental Health Surveys. Journal of Psychosomatic Research 2008; 65: 571-580

5 Ormel J, von Korff M, Burger $H$ et al. Mental disorders among persons with heart disease - results from World Mental Health Surveys. General Hospital Psychiatry 2007; 29: 325-334

6 Scott KM, von Korff M, Ormel J et al. Mental disorders among adults with asthma: results from the World Mental Health Survey. General Hospital Psychiatry 2007; 29: 123-133

7 Härter $M$, Baumeister $H$, Bengel J. Psychische Störungen bei Rehabilitanden mit einer somatischen Erkrankung. In: Härter M, Baumeister $H$, Bengel J, Hrsg. Psychische Störungen bei körperlichen Erkrankungen. Heidelberg: Springer; 2007: 55-69
8 Barth J, Schumacher M, Herrmann-Lingen C. Depression as a risk factor for mortality in patients with coronary heart disease: a meta-analysis. Psychosom Med 2004; 66: 802-813

9 Baumeister H, Härter M. Auswirkungen komorbider psychischer Störungen bei chronischen körperlichen Erkrankungen. Zeitschrift für Medizinische Psychologie 2005; 14: 175-189

10 Hutter N, Knecht A, Baumeister H. Health care costs in persons with asthma and comorbid mental disorders - a systematic review. General Hospital Psychiatry 2011; 33: 443-453

11 Hutter N, Schnurr A, Baumeister H. Healthcare costs in patients with diabetes mellitus and comorbid mental disorders - a systematic review. Diabetologia 2010; 53: 2470-2479

12 DiMatteo MR, Lepper HS, Croghan TW. Depression is a risk factor for noncompliance with medical treatment: Meta-analysis of the effects of anxiety and depression on patient adherence. Arch Intern Med 2000; 160: 2101-2107

13 Ziegelstein RC, Fauerbach JA, Stevens SS et al. Patients with depression are less likely to follow recommendations to reduce cardiac risk during recovery from a myocardial infarction. Arch Intern Med 2000; 160: 1818-1823

14 Baumeister $H$, Hutter $N$, Bengel J et al. Quality of life in medically ill persons with comorbid mental disorders: A systematic review and meta-analysis. Psychotherapy and Psychosomatics 2011; 80: 275-286

15 Härter $M$, Woll S, Reuter $K$ et al. Recognition of psychiatric disorders in musculoskeletal and cardiovascular rehabilitation patients. Archives of Physical Medicine \& Rehabilitation 2004; 85: 1192-1197

16 Irle $H$, Worringen $U$, Korsukéwitz $C$ et al. Erfassung und Behandlung psychischer Beeinträchtigungen in der somatisch-medizinischen Rehabilitation. Rehabilitation 2002; 41: 382-388

17 Reuter $K$, Woll S, Stadelmann $S$ et al. Erkennen und Behandeln psychischer Belastungen und Störungen in der orthopädischen Rehabilitation. Zeitschrift für Klinische Psychologie, Psychiatrie und Psychotherapie 2002; 50: 313-327

18 Rehabilitationswissenschaftlicher Forschungsverbund Freiburg/Bad Säckingen. Empfehlungen für die Umsetzung von Maßnahmen zur Verbesserung der Versorgung psychisch belasteter PatientInnen in der medizinischen Rehabilitation bei somatischen Indikationen. Freiburg/ Bad Säckingen: RFV Freiburg/Bad Säckingen; 2004

19 Jahed J, Kalweit C, Vogel B et al. Implementation and evaluation of a graduated psychodiagnostic procedure in medical rehabilitation centers. Archives of Physical Medicine and Rehabilitation (eingereicht)

20 Jahed J, Bitz I, Bengel J et al. Dokumentation komorbider psychischer Störungen in der medizinischen Rehabilitation: Eine Analyse ärztlicher Entlassungsberichte. Rehabilitation (im Druck)

21 Vogel B, Jahed J, Bengel J et al. Implementierung eines psychodiagnostischen Stufenplans in der medizinischen Rehabilitation: Ergebnisse einer Pilotstudie. Rehabilitation 2009; 48: 361-368

22 Barth J. Ziele und Behandlungserwartungen von psychisch belasteten und depressiven Patienten in der kardiologischen Rehabilitation. Verhaltenstherapie und Verhaltensmedizin 2006; 27: 280-297

23 Baumeister $H$. Behandlungsmotivation. In: Bengel J, Jerusalem M, Hrsg. Handbuch der Gesundheitspsychologie und medizinischen Psychologie. Göttingen: Hogrefe; 2009: 378-386

24 Nübling R, Löschmann C, Hafen $K$ et al. Indication for psychotherapeutic and psychosocial treatment in German rehabilitation. In: Jäckel W, Barth J, Hrsg. Research in rehabilitation: results from a research network in southwest Germany. Stuttgart: Schattauer; 2006: 84-102

25 Paul J, Barth J, Englert $N$ et al. Kardiologische Rehabilitation: Depressive Patienten und ihre Psychotherapiemotivation. In: Petermann F, Ehlebracht-König I, Hrsg. Motivierung, Krankheitsbewältigung und Compliance. Regensburg: Roderer; 2004: 77-88

26 Löwe B, Spitzer RL, Zipfel S et al. PHQ-D. Gesundheitsfragebogen für Patienten. Manual. Karlsruhe: Pfizer; 2002

27 Hermann-Lingen C, Buss U, Snaith RP. HADS-D Hospital Anxiety and Depression Scale - Deutsche Version. Bern: Huber; 2005

28 Hahn D, Reuter K, Härter M. Screening for affective and anxiety disorders in medical patients: comparison of HADS, GHQ-12 and BriefPHQ. GMS Psycho-Social-Medicine 2006; 3: 1-11

29 Reuter $K$, Härter M. Diagnostik psychischer Belastungen und Störungen bei körperlichen Erkrankungen. In: Härter M, Baumeister H, Bengel J, Hrsg. Psychische Störungen bei körperlichen Erkrankungen. Heidelberg: Springer; 2007: 15-28

30 Bergeron E, Poirier L, Fournier LRP et al. Determinants of service use among young Canadians with mental disorders. Canadian Journal of Psychiatry 2005; 50: 629-636

31 Fischer-Kern M, Slunecko T, Leithner $K$ et al. Prädiktoren für die Inanspruchnahme einer Psychotherapie. Nervenarzt 2006; 77: 309-317 
32 Jackson H, Judd F, Komiti A et al. Mental health problems in rural contexts: What are the barriers to seeking help from professional providers? Australian Psychologist 2007; 42: 147-160

33 Wang J, Patten SB, Williams J et al. Help-seeking behaviours of individuals with mood disorders. Canadian Journal of Psychiatry 2005; 50: 652-659

34 Hahn D, Baumeister H, Härter M. Behandlungsmotivation von körperlich kranken Patienten mit komorbiden psychischen Störungen. In: Härter M, Baumeister H, Bengel J, Hrsg. Psychische Störungen bei körperlichen Erkrankungen. Heidelberg: Springer; 2007: 71-82

35 Baumeister H, Krämer L, Brockhaus B. Grundlagen psychologischer Interventionen zur Änderung des Gesundheitsverhaltens. Praxis klinische Verhaltensmedizin und Rehabilitation 2008; 82: 254-264

36 Miller W, Rollnick S. Motivierende Gesprächsführung. Freiburg: Lambertus; 2004

37 Wittchen H, Jacobi F. Die Versorgungssituation psychischer Störungen in Deutschland. Eine klinisch-epidemiologische Abschätzung anhand des Bundes-Gesundheitssurveys 1998. Bundesgesundheitsblatt - Gesundheitsforschung - Gesundheitsschutz 2001; 44: 993-1000

38 Baumeister $H$. Inappropriate prescriptions of antidepressant drugs in patients with subthreshold to mild depression: time for the evidence to become practice. Journal of Affective Disorders 2011, doi:10.1016/j.jad.2011.05.025

39 Baumeister H, Maercker A, Casey P. Adjustment disorders with depressed mood: A critique of its DSM-IV and ICD-10 conceptualization and recommendations for the future. Psychopathology 2009; 42: $139-147$

40 Wittchen $H$, Höfler M, Meister W. Prevalence and recognition of depressive syndromes in German primary care settings: poorly recognized and treated? International Clinical Psychopharmacology 2001; 16: 121-135

41 Leta CR. Untersuchung der Psychotherapiemotivation und deren Einfluss auf die Inanspruchnahme von Psychotherapie. Freiburg i. Br.: Albert-Ludwigs-Universität Freiburg; 2005

42 Maercker A, Enzler A, Grimm G et al. Inanspruchnahme und Psychotherapiemotivation in einer repräsentativen Bevölkerungsstichprobe über 65-Jähriger - Ergebnisse der Zürcher Altersstudie. Psychother Psychosom Med Psychol 2005; 55: 177-182
43 Quinn KM, Laidlaw K, Murray LK. Older peoples' attitude to mental illness. Clinical Psychology and Psychotherapy 2009; 16: 33-45

44 Rabinowitz J, Gross R, Feldmann D. Correlates of a perceived need for mental health assistance and differences between those who do and do not seek help. Social Psychiatry and Psychiatric Epidemiology 1999; 34: 141-146

45 Gatterer G. Multiprofessionelle Altenbetreuung: Ein praxisbezogenes Handbuch. Wien: Springer; 2003

46 Hercek V, Strnad J, Bahro M. Psychotherapie im Alter - Ein Überblick. Krankenhauspsychiatrie 2001; 12: 77-82

47 Heuft G, Kruse A, Radebold H. Lehrbuch der Gerontopsychosomatik und Alterspsychotherapie. München: Reinhard; 2000

48 Maercker A. Alterspsychotherapie und Klinische Gerontopsychologie. Berlin: Springer; 2002

49 Radebold H. Psychoanalytische Psychotherapie und Psychoanalyse im höheren und hohen Erwachsenenalter. Zeitschrift für Psychoanalytische Theorie und Praxis 1994; 9: 439-451

50 Zank S. Einstellungen alter Menschen zur Psychotherapie und Prädiktoren der Behandlungsbereitschaft bei Psychotherapeuten. Verhaltenstherapie und Verhaltensmedizin 2002; 23: 181-194

51 Drapeau A, Lesage A, Boyer $R$. Is the statistical association between sex and the use of services for mental health reasons confounded or modified by social anchorage? Canadian Journal of Psychiatry 2005; 50: 599-604

52 Möller-Leimkühler AM. Barriers to help-seeking by men: a review of sociocultural and clinical literature with particular reference to depression. Journal of Affective Disorders 2002; 71: 1-9

53 Parslow RA, Jorm AF. Who uses mental health services in Australia? An analysis of data from the National Survey of Mental Health and Wellbeing. Australian and New Zealand Journal of Psychiatry 2000; 34: 997-1008

54 Schweickhardt A, Leta R, Bauer J. Inanspruchnahme von Psychotherapie in Abhängigkeit von der Psychotherapiemotivation während der Indikationsstellung in einer Klinikambulanz. Psychother Psychosom Med Psychol 2005; 55: 378-385

55 Albani C, Blaser G, Geyer $M$ et al. Wer nimmt in Deutschland ambulante Psychotherapie in Anspruch? Psychother Psych Med 2009; 59: 281-283 